5-1-2009

\title{
Email: A Note on Hypothesis Tests after Correction for Autocorrelation: Solace for the Cochrane-Orcutt Method?
}

Terry E. Dielman

Texas Christian University, t.dielman@tcu.edu

Follow this and additional works at: http://digitalcommons.wayne.edu/jmasm

Part of the Applied Statistics Commons, Social and Behavioral Sciences Commons, and the Statistical Theory Commons

\section{Recommended Citation}

Dielman, Terry E. (2009) "Email: A Note on Hypothesis Tests after Correction for Autocorrelation: Solace for the Cochrane-Orcutt Method?," Journal of Modern Applied Statistical Methods: Vol. 8 : Iss. 1 , Article 9.

DOI: $10.22237 /$ jmasm/1241136480

Available at: http://digitalcommons.wayne.edu/jmasm/vol8/iss1/9

This Regular Article is brought to you for free and open access by the Open Access Journals at DigitalCommons@WayneState. It has been accepted for inclusion in Journal of Modern Applied Statistical Methods by an authorized editor of DigitalCommons@WayneState. 


\title{
A Note on Hypothesis Tests after Correction for Autocorrelation: Solace for the Cochrane-Orcutt Method?
}

\author{
Terry E. Dielman \\ Texas Christian University
}

The behavior of the $t$ test in small samples for coefficient significance in time-series regressions is examined after using the Prais-Winsten (PW) and Cochrane-Orcutt (CO) corrections for autocorrelation. Results are compared to ordinary least squares and generalized least squares.

Key words: First-order autocorrelation generalized least squares, ordinary least squares, Prais-Winsten, time series regression.

\section{Introduction}

The Prais-Winsten (PW) and Cochrane-Orcutt (CO) methods are popular procedures for correcting for autocorrelation in time-series regression models. Both methods transform the data using a differencing transformation to remove autocorrelation. Ordinary least squares (OLS) applied to the transformed observations will yield estimators that are asymptotically more efficient than OLS applied to the original data.

The PW and CO methods are essentially equivalent except for the treatment of the first observation in the data set. The $\mathrm{CO}$ method simply omits the first observation, while the PW method transforms the observation and retains it. Asymptotically, there is no difference in the efficiency of estimators produced by the two methods. In previous studies of small sample behavior, however, the superior performance of the PW procedure has been documented. Using the $\mathrm{CO}$ procedure results in estimators that are less efficient in small samples. Under certain conditions, the $\mathrm{CO}$ estimator can even be less efficient than OLS applied to the original data.

Terry E. Dielman is a Professor in the M.J. Neeley School of Business, Department of Information Systems and Supply Chain Management. Email: t.dielman@tcu.edu.
Due to the inefficiency of the CO estimator, comparisons of hypothesis testing results from models estimated by PW and CO have not been considered. This article examines the behavior of the $t$ test in small samples for coefficient significance in time-series regressions. Tests are compared using four estimation procedures: OLS, CO, PW and generalized least squares estimation (GLS) using the true value of the autocorrelation coefficient.

The results suggest that the $\mathrm{PW}$ and $\mathrm{CO}$ methods perform similarly when testing hypotheses, but in certain cases, $\mathrm{CO}$ outperforms PW. This does not, however, mean that either method performed particularly well. Both had levels of significance that were much higher than desirable in certain circumstances. The poor performance of these procedures in situations when they are intended to correct for autocorrelation suggests the need for either better estimates of the autocorrelation coefficient, better procedures for correcting for autocorrelation, or alternative approaches that will result in improved hypothesis tests.

\section{Methodology}

The following simple regression model is considered:

$$
y_{t}=\beta_{0}+\beta_{1} x_{t}+\varepsilon_{t} \text { with } \varepsilon_{t}=\rho \varepsilon_{t-1}+\eta_{t}
$$

for $t=1,2, \ldots, T$. In equation (1), $y_{t}$ and $x_{t}$ are the $\mathrm{t}^{\text {th }}$ observations on the dependent and 
explanatory variables, respectively, and $\varepsilon_{t}$ is a random disturbance for the $\mathrm{t}^{\text {th }}$ observation and may be subject to autocorrelation. The $\eta_{t}$ represents disturbance components that are assumed to be independent and identically distributed. The parameters $\beta_{0}$ and $\beta_{1}$ are unknown and must be estimated. The parameter $\rho$ is the autocorrelation coefficient, with $|\rho|<1$. Using matrix notation, the model can be written as:

$$
\mathbf{Y}=\mathbf{X} \boldsymbol{\beta}+\boldsymbol{\varepsilon}
$$

where

$\mathbf{Y}=\left[\begin{array}{c}\boldsymbol{y}_{1} \\ \boldsymbol{y}_{2} \\ \cdot \\ \cdot \\ \cdot \\ \boldsymbol{y}_{\boldsymbol{T}}\end{array}\right], \mathbf{X}=\left[\begin{array}{cc}1 & \boldsymbol{x}_{1} \\ 1 & \boldsymbol{x}_{2} \\ \cdot & \cdot \\ \cdot & \cdot \\ \cdot & \cdot \\ 1 & \boldsymbol{x}_{\boldsymbol{T}}\end{array}\right], \boldsymbol{\varepsilon}=\left[\begin{array}{c}\varepsilon_{1} \\ \varepsilon_{2} \\ \cdot \\ \cdot \\ \cdot \\ \varepsilon_{T}\end{array}\right]$, and $\boldsymbol{\beta}=\left[\begin{array}{c}\beta_{0} \\ \beta_{1}\end{array}\right]$

Two procedures to correct for autocorrelation are examined. These are the Prais-Winsten (1954) and Cochrane-Orcutt (1949) procedures. Both procedures transform the data using the autocorrelation coefficient, $\rho$, after which the transformed data are used in estimation. The procedures differ in their treatment of the first observation, $\left(x_{1}, y_{l}\right)$. The PW transformation matrix is:

$$
\mathbf{M}_{\mathbf{P W}}=\left[\begin{array}{ccccccc}
\sqrt{1-\rho^{2}} & 0 & . & . & . & 0 & 0 \\
-\rho & 1 & . & . & . & 0 & 0 \\
\cdot & \cdot & \cdot & & & \cdot & \cdot \\
\cdot & \cdot & & . & & . & \cdot \\
\cdot & . & & & . & & \\
0 & 0 & . & . & . & -\rho & 1
\end{array}\right]
$$

Pre-multiplying the model in (2) by $\mathbf{M}_{\mathrm{PW}}$ yields

or

$$
\mathbf{M}_{\mathrm{PW}} \mathbf{Y}=\mathbf{M}_{\mathrm{PW}} \mathbf{X} \boldsymbol{\beta}+\mathbf{M}_{\mathrm{PW}} \boldsymbol{\varepsilon}
$$

$$
\mathbf{Y}^{*}=\mathbf{X}^{*} \boldsymbol{\beta}+\boldsymbol{\eta}
$$

where $\mathbf{Y}^{*}$ contains the transformed dependent variable values and $\mathbf{X}^{*}$ is the matrix of transformed independent variable values, so

$$
\mathbf{Y}^{*}=\left\lfloor\sqrt{1-\rho^{2}} y_{1}, y_{2}-\rho y_{1}, \ldots, y_{T}-\rho y_{T-1}\right\rfloor
$$

and

$$
\mathbf{X}^{*}=\left[\begin{array}{cc}
\sqrt{1-\rho^{2}} & \sqrt{1-\rho^{2}} x_{1} \\
1-\rho & \boldsymbol{x}_{2}-\rho \boldsymbol{x}_{1} \\
\cdot & \cdot \\
\cdot & \cdot \\
\cdot & \cdot \\
1-\rho & x_{T}-\rho \boldsymbol{x}_{T-1}
\end{array}\right]
$$

In (6), $\boldsymbol{\eta}$ is the vector of serially uncorrelated $\eta_{t}$ errors.

The $\mathrm{CO}$ transformation matrix is the $(T-1) \times 1$ matrix obtained by removing the first row of the $\mathbf{M}_{\mathrm{PW}}$ transformation matrix. The use of the $\mathrm{CO}$ transformation means that $(T-1)$ observations, rather than $T$, are used to estimate the model. In the $\mathrm{CO}$ transformation, the first observation is omitted, whereas it is transformed and included in the estimation in the PW transformation. Asymptotically, the loss of this single observation is probably of minimal concern. However, for small samples, omitting the first observation has been shown to result in an estimator inferior to that obtained when the first observation is retained and transformed. See Dielman \& Pfaffenberger (1984), Maeshiro (1979), and Park \& Mitchell (1980) for simulation studies demonstrating the efficiency gains of PW, and Doran (1981), Magee (1987), Taylor (1981), and Thornton (1987) for analytical results.

In practice the value of $\rho$ will be unknown and it must be estimated from sample data. The estimators of $\rho$ used will be as follows:

$$
\hat{\rho}_{P W}=\frac{\sum_{t=2}^{T} \hat{\varepsilon}_{t} \hat{\varepsilon}_{t-1}}{\sum_{t=2}^{T} \hat{\varepsilon}_{t}^{2}}
$$




\section{HYPOTHESIS TESTS AFTER CORRECTION FOR AUTOCORRELATION}

when all $T$ observations are used, and

$$
\hat{\rho}_{C O}=\frac{\sum_{t=2}^{T} \hat{\varepsilon}_{t} \hat{\varepsilon}_{t-1}}{\sum_{t=1}^{T-1} \hat{\varepsilon}_{t}^{2}}
$$

when $T-1$ observations are used, where the $\hat{\varepsilon}_{t}$ represent OLS residuals. Park and Mitchell (1980) showed that these two estimators minimize the error sum of squares conditional on $\beta$ when $T$ and $T-1$ observations are used, respectively, in the estimation process.

The actual estimation procedures for both PW and CO are iterative procedures. OLS is run to obtain estimates of the regression coefficients and, subsequently, the $\hat{\varepsilon}_{t}$. The estimator of the autocorrelation coefficient, $\rho$, is computed, the data are transformed, and new estimates of the regression coefficients are obtained. The autocorrelation coefficient estimate is recomputed and compared to the previous estimate. In the results, if these estimates differ by less than 0.000001 , the iterative procedure stops. The procedure also stops when it reaches 25 iterations. If boundary conditions are encountered the estimate of $\rho$ is set at \pm 0.999999 .

The model considered in this article is described in equation (1). The explanatory variable values are generated as follows:

1. $x_{t}=\lambda x_{t-1}+u_{t}$ for $t=1,2, \ldots, T$ with the $u_{\mathrm{t}}$ chosen from the $\mathrm{N}(0,2)$ distribution. The values of $\lambda$ used were $0.0,0.4$ and 0.8 .

2. A stochastic time trend is used. In this case $x_{t}=\lambda t+u_{t}$ for $t=1,2, \ldots, T$ and $u_{\mathrm{t}}$ is chosen from the $\mathrm{N}(0,2)$ distribution for $\lambda=0.4$ and 0.8 .

Once generated, these values are held fixed throughout the experiment for each sample size. The disturbances, $\eta_{t}$, are chosen from the $\mathrm{N}(0,1)$ distribution. After generating the $\eta_{\mathrm{t}}$, the $\varepsilon_{\mathrm{t}}$ values are created as $\varepsilon_{t}=\rho \varepsilon_{t-1}+\eta_{t}$ where $\varepsilon_{0}=\frac{\eta_{0}}{1-\rho^{2}}$ and $\eta_{0}$ is an initial draw from the disturbance distribution. The explanatory variable values were generated independently of the disturbances.

The parameter $\beta_{0}$ was set equal to zero (without loss of generality). The parameter $\beta_{1}$ was set equal to zero to examine the level of significance. For each factor combination in the experimental design, ten thousand Monte Carlo trials were used to assess levels of significance. A sample size of $T=20$ was used. The values of $\rho$ were $0.0,0.2,0.4,0.6,0.8$, and 0.95 . The null hypothesis $\mathrm{H}_{0}: \beta_{1}=0$ is tested using the $t$ test and the number of rejections of the null hypothesis was recorded to assess the level of significance.

The hypothesis tests were also conducted with $\beta_{1}=0.2,0.3,0.4,0.5$, and 1.0. When $\mathrm{H}_{0}$ is rejected, the proportion of correct rejections can be used to construct empirical power functions. Power comparisons based on the original simulations are complicated by the differences in the observed significance levels. A valid power comparison can be made only if the true significance levels of the tests are similar, which is clearly not the case based on our results.

The power comparison was accomplished using a procedure suggested by Zhang and Boos (1994). From the original 10,000 simulations with $\beta_{1}=0$, the test statistics were sorted and the critical values producing a $5 \%$ level of significance were chosen for each design point. These values represent estimates of the critical values under the null hypothesis that produce an exact $5 \%$ level of significance. The simulation was repeated with the non-zero values of $\beta_{1}$, using the empirically determined critical values. The test statistics from the second set of simulations will have similar levels of significance, making their powers comparable. Zhang and Boos (1994) suggested using a larger number of Monte Carlo trials to estimate the correct critical value under the null hypothesis if possible. In this experiment 10,000 trials under the null and 5,000 under the alternative hypotheses were used.

Results are reported for four estimation procedures: OLS (assuming $\rho=0$ ), PW and CO and GLS (which is the PW procedure using the true value of $\rho$ ). All random numbers were 
generated using IMSL subroutines and the simulation was written in FORTRAN.

\section{Results}

Consider Tables 1 and 2. These tables show the number of rejections of the true null hypothesis that the slope is zero for all factor combinations in the Monte Carlo simulation. Table 1 shows the results for the autoregressive independent variable; Table 2 for the stochastic trend variable. The most striking results are for the autoregressive case when $\lambda$ is 0.8 and the stochastic trend case for $\lambda$ equal to both 0.4 and 0.8 . As the level of autocorrelation increases, the observed levels of significance become very high for OLS, but this is not unexpected. OLS is not expected to perform well when disturbances are autocorrelated.

However, the two methods that correct for autocorrelation do not perform well either. PW has very high rejection rates with some cases approaching $50 \%$. The rejection rates for $\mathrm{CO}$ are high as well, but often not as high as PW. This is particularly evident when the independent variable is autoregressive. These results suggest that correcting for autocorrelation does not guarantee reliable inferences about the slope coefficient.

Selected power comparisons using 5,000 Monte Carlo trials are shown in Table 3 for the autoregressive independent variable with $\lambda=0.8$ and in Table 4 for the stochastic trend variable with $\lambda=0.8$. When the independent variable is autoregressive, $\mathrm{CO}$ generally has power equal to or slightly higher than PW. Figures 1 and 2 plot the empirical power curves for $\rho=0.0$ and $\rho=$ 0.95 from Table 3. When $\rho=0.0$ there is little difference in adjusted power; when $\rho=0.95 \mathrm{CO}$ has higher power than PW.

When the independent variable is a stochastic trend, there is little difference between $\mathrm{PW}$ and $\mathrm{CO}$ as evidenced in the empirical power curves in Figures 3 and 4 for $\rho=0.0$ and $\rho=$ 0.95 , respectively. In this case, when $\rho=0.95$, it is especially troublesome that OLS has higher adjusted power than either PW or $\mathrm{CO}$, which supposedly adjust for autocorrelation. This result is driven by the very high levels of significance for OLS of course.

\section{Conclusion}

Previous studies have shown that the PW method is superior to $\mathrm{CO}$ as a correction for autocorrelation in terms of estimator efficiency. However, these results do not hold up in an examination of inference results. $\mathrm{CO}$ generally performs as well and in many cases better than PW in terms of observed level of significance and adjusted power. This should not be taken as a suggestion that the PW method should be abandoned and $\mathrm{CO}$ resorted to, however. Perhaps both methods should be abandoned and a better approach sought for handling autocorrelation in regression models. In terms of inference, a bootstrap approach as Rayner (1991) suggested might be preferred to either the PW or CO method. Alternatively, as suggested by Mizon (1995), perhaps another approach to correcting for autocorrelation should be considered. Bayesian estimators (see Ohtani, 1990, and Kennedy \& Simons, 1991) also hold promise for improvements.

\section{References}

Cochrane, D., \& Orcutt, G. (1949). Application of least squares regression to relationships containing autocorrelated error terms. Journal of the American Statistical Association, 44, 32-61.

Dielman, T., \& Pfaffenberger, R. (1984). Small sample properties of estimators in the autocorrelated error model: A review and some additional simulations. Statistical Papers/Statistiche Hefte, 30, 163-183.

Doran, H. (1981). Omission of an observation from a regression analysis: A discussion on efficiency loss with applications. Journal of Econometrics, 16, 367-374.

Kennedy, P., \& Simons, D. (1991). Fighting the teflon factor: Comparing classical and Bayesian estimators for autocorrelated errors. Journal of Econometrics, 48, 15-27.

Maeshiro, A. (1979). On the retention of the first observation in serial correlation adjustment of regression models. International Economic Review, 20, 259-265.

Magee, L. (1987). A note on CochraneOrcutt estimation. Journal of Econometrics, 35, 211-218. 
Table 1: Empirical Significance Level: Number of Rejections of True Null Hypothesis $\mathrm{H}_{0}$ : $\beta_{1}=$ 0 Using Autoregressive Independent Variable (10,000 Trials)

\begin{tabular}{|c|c|c|c|c|c|c|}
\hline \multirow{2}{*}{ Lambda $=0.0$} & 0.00 & 0.20 & 0.40 & 0.60 & 0.80 & 0.95 \\
\hline OLS & 508 & 601 & 623 & 531 & 342 & 226 \\
\hline PW & 754 & 757 & 716 & 637 & 523 & 462 \\
\hline GLS & 508 & 500 & 516 & 514 & 526 & 530 \\
\hline CO & 699 & 716 & 697 & 617 & 471 & 424 \\
\hline Lambda $=0.4$ & 0.00 & 0.20 & 0.40 & 0.60 & 0.80 & 0.95 \\
\hline OLS & 516 & 712 & 870 & 852 & 700 & 491 \\
\hline PW & 808 & 819 & 826 & 755 & 640 & 546 \\
\hline GLS & 516 & 516 & 501 & 503 & 508 & 493 \\
\hline CO & 744 & 771 & 764 & 694 & 566 & 447 \\
\hline Lambda $=0.8$ & 0.00 & 0.20 & 0.40 & 0.60 & 0.80 & 0.95 \\
\hline OLS & 521 & 865 & 1307 & 1848 & 2658 & 3613 \\
\hline PW & 806 & 942 & 1048 & 1232 & 1442 & 1679 \\
\hline GLS & 521 & 512 & 512 & 506 & 505 & 496 \\
\hline CO & 726 & 877 & 995 & 1057 & 949 & 822 \\
\hline
\end{tabular}

Table 2: Empirical Significance Level: Number of Rejections of True Null Hypothesis $\mathrm{H}_{0}: \beta_{1}=0$ Using Stochastic Trend Independent Variable (10,000 Trials)

\begin{tabular}{|c|c|c|c|c|c|c|}
\hline \multirow{2}{*}{ Lambda $=0.4$} & 0.00 & 0.20 & 0.40 & 0.60 & 0.80 & 0.95 \\
\hline OLS & 453 & 936 & 1685 & 2848 & 4537 & 6111 \\
\hline PW & 842 & 1026 & 1291 & 1815 & 2965 & 4496 \\
\hline GLS & 453 & 458 & 475 & 482 & 477 & 494 \\
\hline CO & 805 & 1008 & 1307 & 1860 & 2955 & 4419 \\
\hline Lambda $=0.8$ & 0.00 & 0.20 & 0.40 & 0.60 & 0.80 & 0.95 \\
\hline OLS & 467 & 1013 & 1822 & 3087 & 4812 & 6352 \\
\hline PW & 831 & 1039 & 1347 & 1941 & 3181 & 4711 \\
\hline GLS & 467 & 456 & 453 & 459 & 484 & 487 \\
\hline CO & 819 & 1036 & 1399 & 2038 & 3288 & 4706 \\
\hline
\end{tabular}


Table 3: Adjusted Power Comparisons Using Autoregressive Independent Variable with Lambda $=0.8$ (5,000 trials)

\begin{tabular}{|c|c|c|c|c|c|c|}
\hline \multirow{2}{*}{$\mathrm{Rho}=0.0$} & \multicolumn{6}{|c|}{ Beta $=$} \\
\hline & 0.0 & 0.2 & 0.3 & 0.4 & 0.5 & 1.0 \\
\hline OLS & 250 & 3006 & 4572 & 4961 & 4998 & 5000 \\
\hline PW & 250 & 3107 & 4535 & 4922 & 4988 & 5000 \\
\hline GLS & 250 & 3006 & 4572 & 4961 & 4998 & 5000 \\
\hline $\mathrm{CO}$ & 250 & 3108 & 4550 & 4920 & 4992 & 5000 \\
\hline \multirow{2}{*}{ Rho $=0.2$} & \multicolumn{6}{|c|}{ Beta $=$} \\
\hline & 0.0 & 0.2 & 0.3 & 0.4 & 0.5 & 1.0 \\
\hline OLS & 250 & 2476 & 4079 & 4819 & 4976 & 5000 \\
\hline $\mathrm{PW}$ & 250 & 2332 & 3856 & 4695 & 4944 & 5000 \\
\hline GLS & 250 & 2361 & 4043 & 4817 & 4978 & 5000 \\
\hline $\mathrm{CO}$ & 250 & 2457 & 3991 & 4731 & 4953 & 5000 \\
\hline \multirow{2}{*}{$\mathrm{Rho}=0.4$} & \multicolumn{6}{|c|}{ Beta $=$} \\
\hline & 0.0 & 0.2 & 0.3 & 0.4 & 0.5 & 1.0 \\
\hline OLS & 250 & 2039 & 3407 & 4429 & 4845 & 5000 \\
\hline $\mathrm{PW}$ & 250 & 1737 & 3003 & 4175 & 4749 & 5000 \\
\hline GLS & 250 & 1860 & 3420 & 4507 & 4907 & 5000 \\
\hline $\mathrm{CO}$ & 250 & 1932 & 3254 & 4346 & 4795 & 5000 \\
\hline \multirow{2}{*}{$\mathrm{Rho}=0.6$} & \multicolumn{6}{|c|}{ Beta $=$} \\
\hline & 0.0 & 0.2 & 0.3 & 0.4 & 0.5 & 1.0 \\
\hline OLS & 250 & 1684 & 2711 & 3659 & 4364 & 5000 \\
\hline PW & 250 & 1286 & 2313 & 3359 & 4252 & 5000 \\
\hline GLS & 250 & 1526 & 2925 & 4120 & 4729 & 5000 \\
\hline $\mathrm{CO}$ & 250 & 1655 & 2771 & 3749 & 4490 & 5000 \\
\hline \multirow{2}{*}{$\mathrm{Rho}=0.8$} & \multicolumn{6}{|c|}{ Beta $=$} \\
\hline & 0.0 & 0.2 & 0.3 & 0.4 & 0.5 & 1.0 \\
\hline OLS & 250 & 1478 & 2153 & 2849 & 3475 & 4906 \\
\hline PW & 250 & 1089 & 1808 & 2656 & 3497 & 4967 \\
\hline GLS & 250 & 1424 & 2771 & 3935 & 4637 & 5000 \\
\hline $\mathrm{CO}$ & 250 & 1651 & 2430 & 3287 & 3970 & 4977 \\
\hline \multirow{2}{*}{ Rho $=0.95$} & \multicolumn{6}{|c|}{ Beta $=$} \\
\hline & 0.0 & 0.2 & 0.3 & 0.4 & 0.5 & 1.0 \\
\hline OLS & 250 & 1430 & 1874 & 2361 & 2852 & 4473 \\
\hline PW & 250 & 973 & 1526 & 2235 & 3048 & 4821 \\
\hline GLS & 250 & 1532 & 2911 & 4072 & 4686 & 5000 \\
\hline $\mathrm{CO}$ & 250 & 1726 & 2307 & 3025 & 3626 & 4835 \\
\hline
\end{tabular}


HYPOTHESIS TESTS AFTER CORRECTION FOR AUTOCORRELATION

Figure 1: Power Curve for Testing Slope Equal Zero:

Autoregressive With Lambda $=0.8 ;$ rho $=0.0$

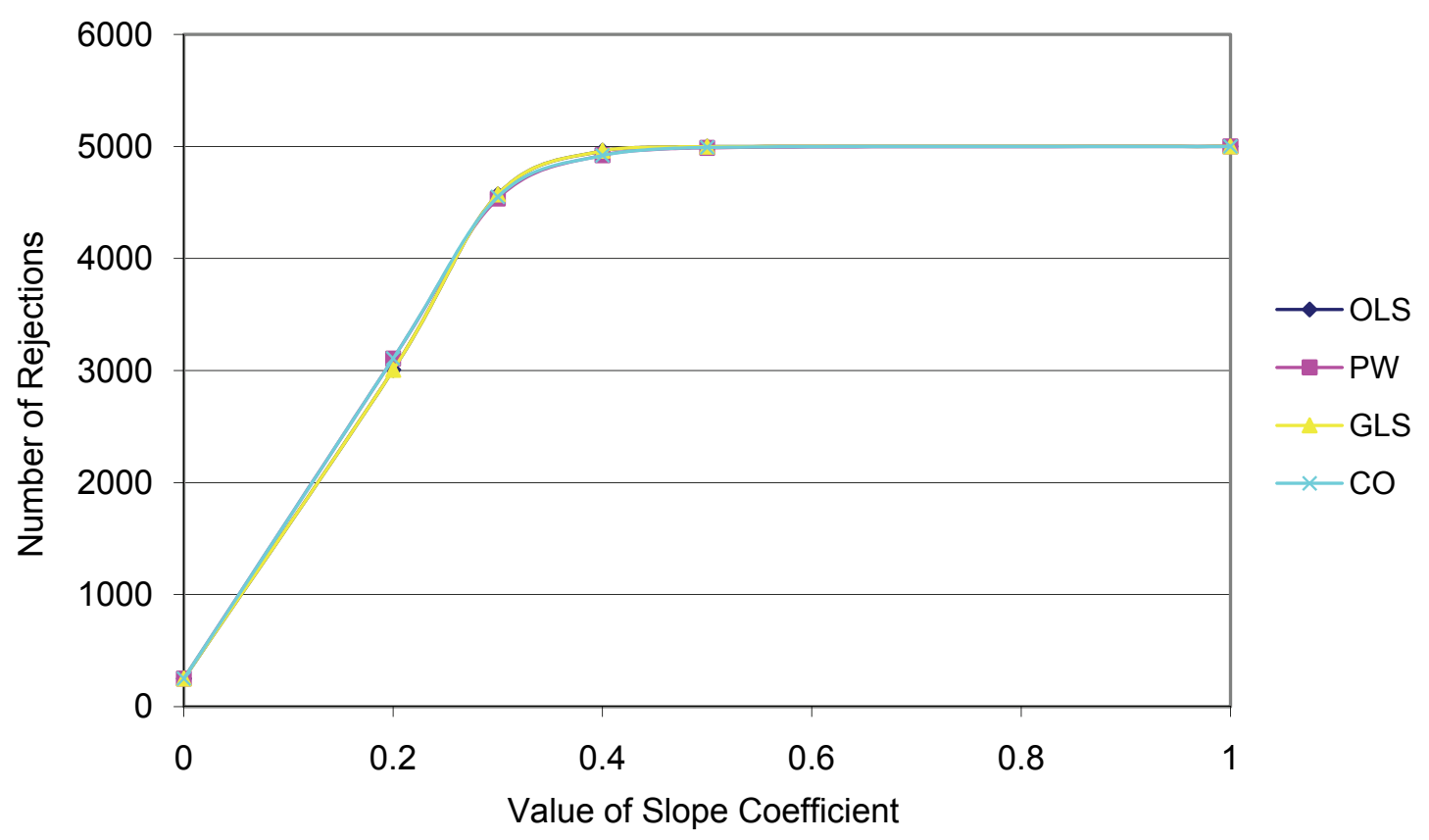

Figure 2: Power Curve for Testing Slope Equal Zero:

Autoregressive With Lambda $=0.8$, rho $=0.95$

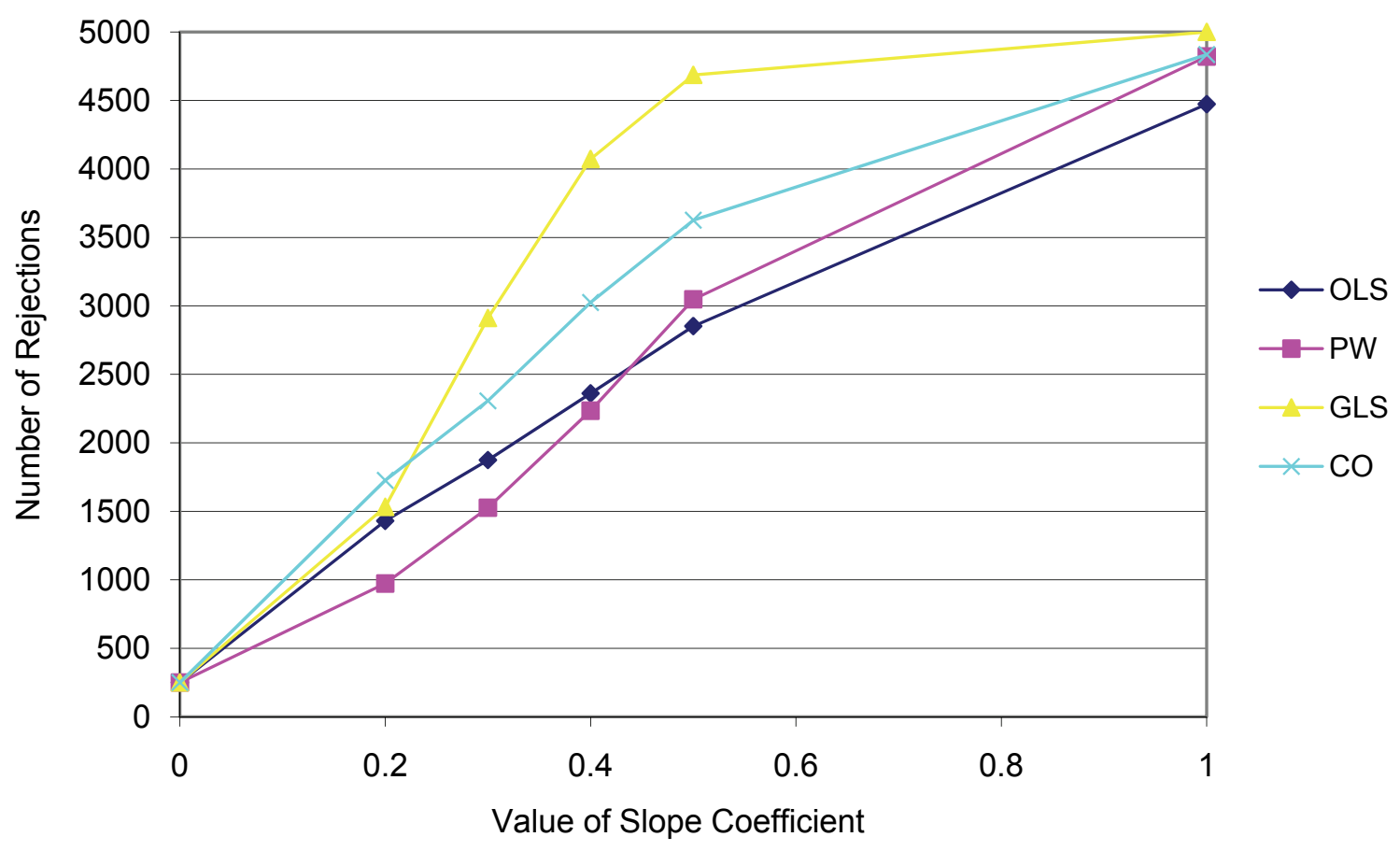




\section{DIELMAN}

Table 4: Adjusted Power Comparisons Using Stochastic Trend Independent Variable with Lambda $=0.8$ (5,000 trials)

\begin{tabular}{|c|c|c|c|c|c|c|}
\hline \multirow{2}{*}{ Rho $=0.00$} & \multicolumn{6}{|c|}{ Beta $=$} \\
\hline & 0.0 & 0.2 & 0.3 & 0.4 & 0.5 & 1.0 \\
\hline OLS & 250 & 4866 & 4999 & 5000 & 5000 & 5000 \\
\hline PW & 250 & 4785 & 4983 & 4998 & 5000 & 5000 \\
\hline GLS & 250 & 4866 & 4999 & 5000 & 5000 & 5000 \\
\hline $\mathrm{CO}$ & 250 & 4666 & 4959 & 4993 & 4996 & 5000 \\
\hline \multirow{2}{*}{ Rho $=0.20$} & \multicolumn{6}{|c|}{ Beta $=$} \\
\hline & 0.0 & 0.2 & 0.3 & 0.4 & 0.5 & 1.0 \\
\hline OLS & 250 & 4475 & 4990 & 5000 & 5000 & 5000 \\
\hline PW & 250 & 4124 & 4873 & 4988 & 5000 & 5000 \\
\hline GLS & 250 & 4494 & 4994 & 5000 & 5000 & 5000 \\
\hline $\mathrm{CO}$ & 250 & 3900 & 4788 & 4959 & 4989 & 5000 \\
\hline \multirow{2}{*}{ Rho $=0.40$} & \multicolumn{6}{|c|}{ Beta $=$} \\
\hline & 0.0 & 0.2 & 0.3 & 0.4 & 0.5 & 1.0 \\
\hline OLS & 250 & 3591 & 4817 & 4992 & 5000 & 5000 \\
\hline PW & 250 & 2860 & 4389 & 4869 & 4984 & 5000 \\
\hline GLS & 250 & 3768 & 4882 & 4998 & 5000 & 5000 \\
\hline $\mathrm{CO}$ & 250 & 2656 & 4207 & 4752 & 4931 & 5000 \\
\hline \multirow{2}{*}{ Rho $=0.60$} & \multicolumn{6}{|c|}{ Beta $=$} \\
\hline & 0.0 & 0.2 & 0.3 & 0.4 & 0.5 & 1.0 \\
\hline OLS & 250 & 2268 & 3856 & 4712 & 4959 & 5000 \\
\hline PW & 250 & 1520 & 2963 & 4176 & 4714 & 5000 \\
\hline GLS & 250 & 2673 & 4294 & 4916 & 4996 & 5000 \\
\hline $\mathrm{CO}$ & 250 & 1440 & 2840 & 3964 & 4540 & 4997 \\
\hline \multirow{2}{*}{ Rho $=0.80$} & \multicolumn{6}{|c|}{ Beta $=$} \\
\hline & 0.0 & 0.2 & 0.3 & 0.4 & 0.5 & 1.0 \\
\hline OLS & 250 & 1133 & 2124 & 3209 & 4022 & 5000 \\
\hline PW & 250 & 715 & 1406 & 2295 & 3193 & 4962 \\
\hline GLS & 250 & 1713 & 3143 & 4291 & 4841 & 5000 \\
\hline $\mathrm{CO}$ & 250 & 701 & 1371 & 2232 & 3049 & 4880 \\
\hline \multirow{2}{*}{ Rho $=0.95$} & \multicolumn{6}{|c|}{ Beta $=$} \\
\hline & 0.0 & 0.2 & 0.3 & 0.4 & 0.5 & 1.0 \\
\hline OLS & 250 & 685 & 1161 & 1766 & 2446 & 4661 \\
\hline PW & 250 & 415 & 713 & 1112 & 1608 & 4074 \\
\hline GLS & 250 & 1294 & 2457 & 3634 & 4450 & 5000 \\
\hline $\mathrm{CO}$ & 250 & 442 & 733 & 1164 & 1660 & 4024 \\
\hline
\end{tabular}


HYPOTHESIS TESTS AFTER CORRECTION FOR AUTOCORRELATION

Figure 3: Power Curve for Testing Slope Equal Zero:

Stochastic Trend With Lambda $=0.8 ;$ rho $=0.0$

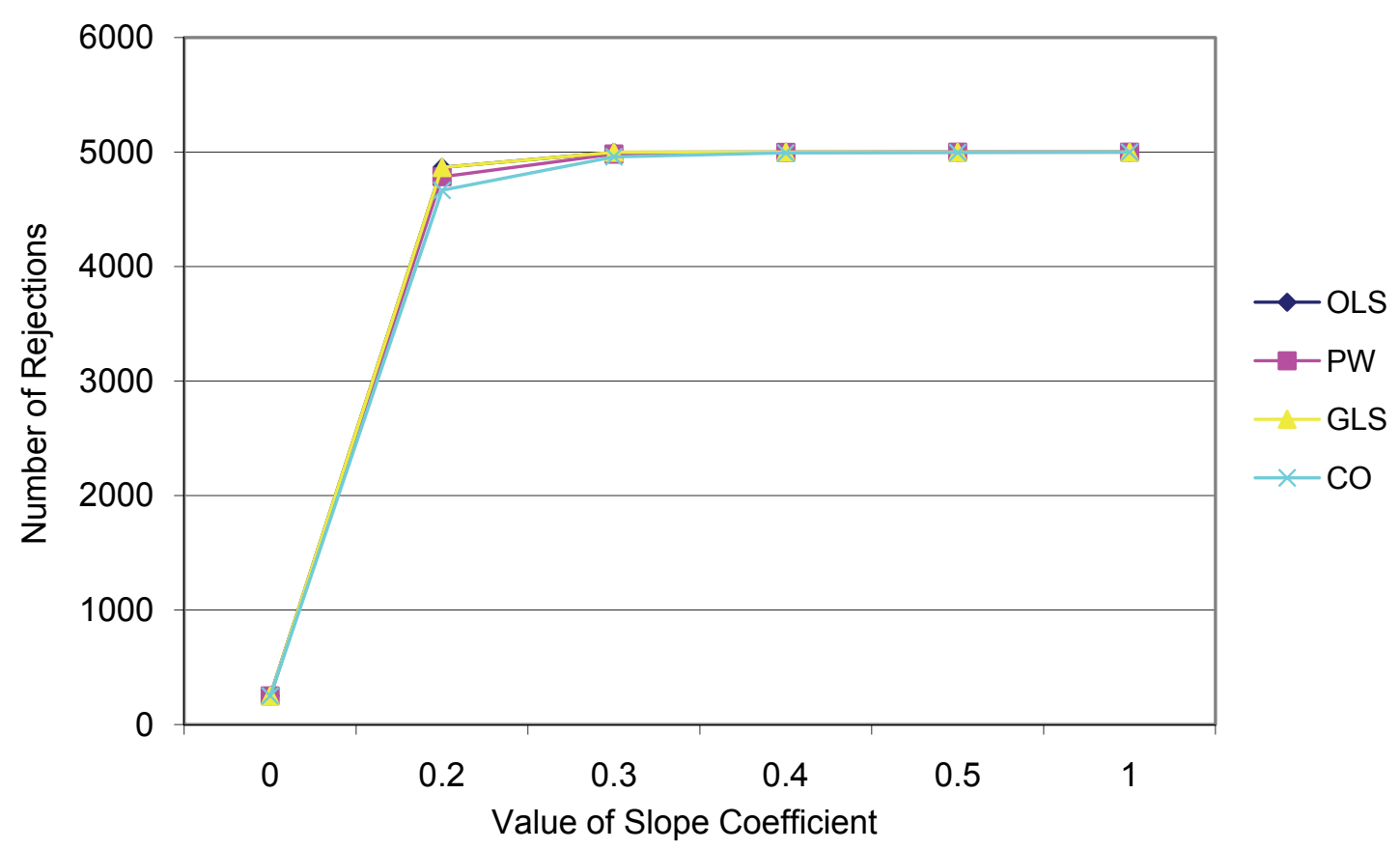

Figure 4: Power Curve for Testing Slope Equal Zero:

Stochastic Trend With Lambda $=0.8 ;$ rho $=0.95$

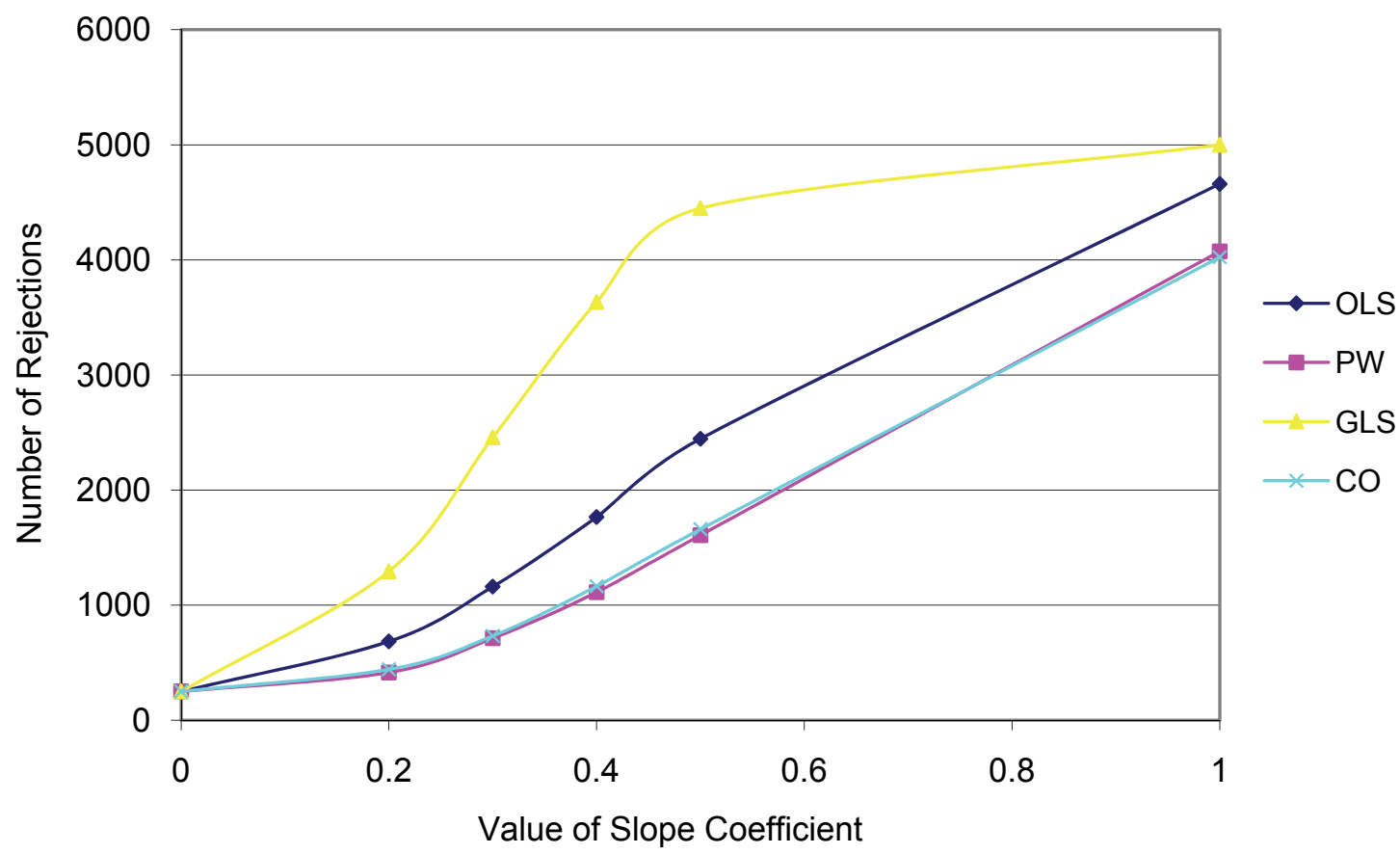


Mizon, G. (1995). A simple message for autocorrelation correctors: Don't. Journal of Econometrics, 69, 267-288.

Ohtani, K. (1990). On estimating and testing in a linear regression model with autocorrelated errors. Journal of Econometrics, 44, 333-346.

Park, R., \& Mitchell, G. (1980). Estimating the autocorrelated error model with trended data. Journal of Econometrics, 13, 185201.

Prais, S., \& Winsten, C. (1954). Trend estimators and serial correlation. Cowles Commission Discussion Paper: Stat. No. 383, Chicago, IL.
Rayner, R. (1991). Resampling methods for tests in regression models with autocorrelated errors. Economics Letters, 36, 281-284.

Taylor, W. (1981). On the efficiency of the Cochrane-Orcutt estimator. Journal of Econometrics, 17, 67-82.

Thornton, D. (1987). A note on the efficiency of the Cochrane-Orcutt estimator of the AR(1) regression model. Journal of Econometrics, 36, 369-376.

Zhang, J., \& Boos, D. (1994). Adjusted power estimates in Monte Carlo experiments. Communication in Statistics - Simulation and Computation, 23, 165-173. 